\title{
An EPQ model under two levels of trade credit and limited storage space
}

\author{
Alok kumar $^{\mathrm{a}^{*}}$, K K Kaanodiya ${ }^{\mathrm{b}}$ and R R Pachauri ${ }^{\mathrm{c}}$ \\ ${ }^{a}$ Institute of Engineering and Technology, Khandhari, Agra, India \\ ${ }^{b}$ Department of Mathematics BSA College Mathura, India \\ ${ }^{c}$ Research Scholar Department of Mathematics, B.S.A. College, Mathura, India

ART I L E I N F O ABSTRACT \\ Article history: \\ Received 25 October 2011 \\ Received in revised form \\ November, 2, 2011 \\ Accepted January, 262012 \\ Available online \\ 28 January 2012 \\ Keywords: \\ EPQ \\ Trade credit \\ Limited storage space \\ Inventory

\begin{abstract}
In this article, a production inventory model is developed to determine the retailer's optimal replenishment decisions under two levels of trade credit and limited storage space. Two levels of trade credit refers that the supplier provides to his/her retailer a permissible delay period to customers credit period $\mathrm{N}$ provides by the retailer. This paper investigates retailer optimal cost. Three theorems are developed to determine optimal cycle time, optimal relevant cost and analyze the validity of propose model, Sensitive analysis shows managerial decisions for retailer.
\end{abstract} \\ settle the account and the retailer also in turn provides a delay period to his/her customer for \\ paying of purchasing goods. The retailer's credit period $\mathrm{M}$ offered by supplier is greater than \\ replenishment policy under finite replenishment rate by minimizing the total annual inventory \\ optimal order quantity for retailers accurate and rapidly. A numerical example is used to
}

(C) 2012 Growing Science Ltd. All rights reserved

\section{Introduction}

Over the years, numerous research articles have been published in different journals on trade credit under various conditions, in which the suppliers offers a delay period to settle the retailers account. The retailer takes the benefit of trade credit and sells the product and earns interest by putting generated revenue in an interest bearing account. The supplier does not charge any interest if the payment is made before credit period. However, if the payment is paid beyond the predefined period, a high interest rate is charged. Many researchers contribute on trade credit with variety of conditions.

Goyal (1985) is believed to be the first who developed the economic order quantity (EOQ) model under the conditions of permissible delay in payments. Davis and Gaither (1985) presented EOQ model when supplier offers one time opportunity to delay the payments of order in case an order for additional units is placed. Chand and Ward (1987) investigated Goyal (1985) model under the assumptions of classical economic order quantity model. Shah et al. (1988) studied the same model incorporating shortages. Mondal and Phaujdar (1989c) extended this issue by considering the interest earned from the sales revenue. Shah (1993a, b) also developed EOQ models for perishable items where delay in payment is permissible. Aggarwal and Jaggi (1995) developed Goyal's (1985) model

* Corresponding author.

E-mail: kkkaanodiya@yahoo.in and rrpachauri@gmail.com (R R Pachauri)

(C) 2012 Growing Science Ltd. All rights reserved. doi: $10.5267 /$ j.ijiec.2012.01.005 
to the case of exponential deterioration, Shah and Sreehari (1996) presented an extended issue of EOQ model where the delay in payment is permitted and the capacity of own warehouse is limited. Liao (2000) and Sarker (2000a) deal this problem with inflation. Jamal et al. (1997) and Chang and Dye (2001) examined this issue with allowable shortage. Chang et al. (2001) investigated this topic in the case of linear trend demand. Mondal and Phaujdar (1989b), Hwang and Shinn (1997), Jamal et al. (1997) and Sarker et al. (2000b) extended this issue with deteriorating items. Teng (2002) modified Goyal's (1985) model by assuming the selling price is not necessary equal to purchase price. Other papers related to this area are Chang and Dye (2002), Chang el al. (2001), Chen and Chung (1999), Chung (1998a, b) Jamal et al. (2000), Sarker et al. (2000a), Salameh et al. (2003), Huang and Chung (2003), Arcelus et al. (2003), Abad and Jaggi (2003), Shinn and Huang (2003), Chung and Huang (2003), Chung and Liao (2004), Chung et al. (2005), Chung and Liao (2006), Huang (2007a) and Liao and Chung (2009).

In above models, the supplier adopts a business strategy of permissible delay period in paying the purchasing cost to attract more customers. The retailer takes the benefit of trade credit and sells the product and earns the interest by putting generated revenue in an interest bearing account. They implicitly assumed that the buyer would pay the supplier as soon as he/she receives the items. As mentioned earlier, supplier only offers a trade credit to the retailer but retailer does not provide any trade credit to his/her customer which means we deal with one level of trade credit. However, in many real-world cases, this condition will not hold. Recently, Huang (2003) modified this assumption by assuming that the retailer will also adopt the trade credit policy to stimulate his/her customer demand to develop retailer replenishment model and this method is called two level of trade credit policy. Retailers take benefits of trade credit and place an order of large quantity of goods to supplier to reduce inventory cost. Since retailer's storage capacity is limited so he/she has to take a rented warehouse for excess quantities to store his goods. This paper is strongly related for small and medium size retailers whose storage capacity is limited especially the growing markets like in India. When retailer receives an order the delivery is first replenishment from rented warehouse because holding cost of rented warehouse is higher with the respect of own warehouse. Numerous researchers have focused in this area such as Sharma (1987), Pakkala and Achary (1992), Benkerouf (1997), Goswami and Chaudhuri (1992), Bunia and Maiti (1998), Huang et al. (2006) and Lee and Hsu (2009) and much more. Huang (2007b) extended Chung and Huang (2003) and Huang (2003) model in the light of two level of trade credit and determine retailer's replenishment decision with EPQ framework. Teng and Chang (2009) extended Huang (2007b) and Teng and Goyal (2007) model in more realistic situation in which the retailer obtains its revenue from $N$ to $T+N$ not from 0 to $T$ and $N<M$.

This paper extends Huang (2006) model by assuming finite replenishment. We developed a mathematical model to determine optimal retailer's replenishment policy under two levels of trade credit and limited storage space in EPQ framework. Three theorems are developed to accurate and rapidly determine optimal cycle time, optimal relevant cost and optimal order quantity for retailers under minimizing total cost of inventory system. Finally, a numerical example explore the validity of propose model.

\section{Assumptions and Notations}

For convenience, most assumptions and notations throughout in this paper used Huang (2006) model.

\subsection{Assumptions}

1) Demand rate is known and constant

2) Shortages are not allowed.

3) Time horizon is infinite. 
4) Replenishment is finite, known and constant

5) $s \geq c, k \geq h, I_{p} \geq I_{e}$ and $M \geq N$.

6) If the orders quantity is larger than retailer's OW storage capacity $\mathrm{W}$, the excess quantity is stored in rented warehouse. Since, storage capacity of rented warehouse is unlimited. When orders received by retailer the items is first replenishment from rented warehouse.

7) Before settling the account the retailer's takes the benefit of trade credit and sells the product and earn the interest by putting generated revenue in an interest bearing account. When the cycle time $(T)$ is greater than or equal to the retailer's credit period $(M)$ offered by the supplier. Then, the retailer settles the account at $T=M$. The retailer pays for the product sold and keep his/her profit and start paying interest on unsold items at rate $I_{P}$. When the cycle time $(T)$ is less than or equal to the retailer's credit period $(M)$, the retailer settles the account at $T=M$. Therefore, the retailer does not have to pay any interest charges.

8) The retailer can accumulate revenue and earn interest when his/her customer pays the amount for purchasing goods to the retailer by the end of predefined trade credit period offered by supplier. The retailer earns the interest by putting generated revenue in an interest bearing account at the rate of $I e$ per unit per year; i.e. the retailer can earn interest during the period $[N, M]$ with rate $I e$ under the condition of trade credit.

9) The transportation cost between own warehouse and rented warehouse is negligible.

\subsection{Notation}

1) $A$ Ordering cost per order

2) $c$ Purchasing price per item

3) $D$ Demand rate per year

4) $P$ Replenishment rate per year. $P \geq D$ (Decision variable)

5) $\rho 1-\frac{D}{P} \geq 0$

6) $h$ OW stock-holding cost per item per year

7) $I_{e}$ Interest earned per $\$$ per year

8) $I_{p}$ Interest charged per $\$$ per year

9) $k$ RW stock-holding cost per item per year (Decision variable)

10) $M$ The retailer's trade credit period offered by supplier in years $(M>N)$

11) $N$ The customer's trade credit period offered by retailer in years (Decision variable)

12) $s$ Selling price per item (Decision variable)

13) $t_{w}$ The rented warehouse time in years, $t_{w}= \begin{cases}\frac{D T \rho-W}{D} & \text { ifDT } \rho>W\left(T>\frac{W}{D \rho}\right) \\ 0 & \text { ifDT } \rho<W\left(T<\frac{W}{D \rho}\right)\end{cases}$

14) $w$ Retailer's OW storage capacity

15) $\operatorname{TRC}(T)$ The annual total relevant cost which is a function of $T$ (Decision variable)

16) $T^{*}$ The optimal cycle time of $\operatorname{TRC}(T)$ (Decision variable)

\section{The Model}

The total annual relevant cost of inventory system consists of the following elements. Three situations may arise $\quad$ (1) $M \geq N \geq \frac{W}{D \rho}$, (2) $M \geq \frac{W}{D \rho} \geq N$ and (3) $\frac{W}{D \rho} \geq M \geq N$.

Case. 1 Suppose that $M \geq N \geq W / D \rho$

(1) Annual ordering cost $=\frac{A}{T}$ 
(2) According to assumption (6) annual stock-holding cost (excluding interest charges ) can be obtain as follow:

1. $W / D \rho<T$

In this case the order quantity larger than retailer's OW storage capacity. So the retailer needs to rent the warehouse to storage the exceeding items. See in Fig. 1 Hence

Annual stock holding cost $=$ annual stock holding cost of rented warehouse + annual stock-holding cost of the storage capacity $W$

$$
=\frac{K t_{W}(D T \rho-W)}{2 T}+\frac{h W[D T(1+\rho)-W]}{2 D T}=\frac{K(D T \rho-W)^{2}}{2 D T}+\frac{h W[D T(1+\rho)-W]}{2 D T}
$$

\section{2. $T \leq W / D \rho$}

In this case order quantity is not larger than retailer's storage capacity. So the retailer will not necessary to rent warehouse to storage items. Hence

Annual stock-holding cost $=\frac{D T h \rho}{2}$

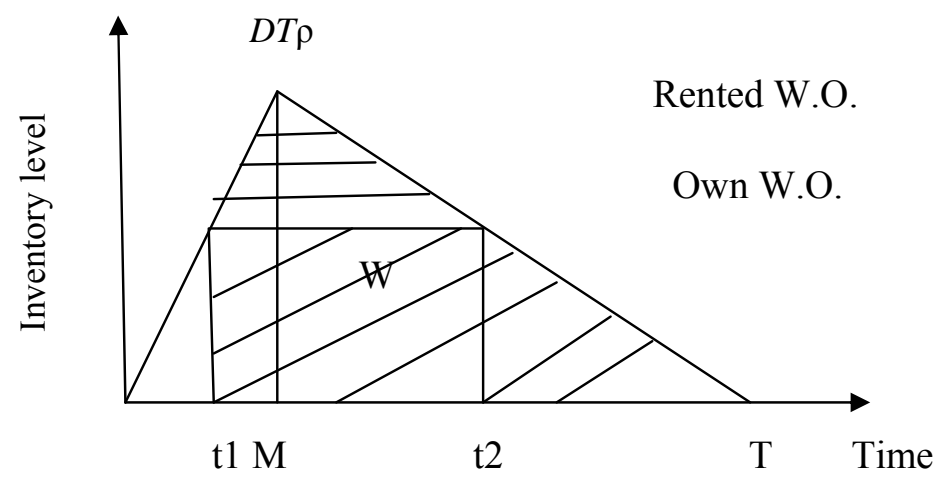

Fig. 1. The holding cost of inventory in Rented W.O. and Own W.O.

(3) According to assumption (7) cost of interest charges for the items kept in stock per year can be obtained as follows:

1. $M \leq \frac{P M}{D} \leq T$

In this case, interest charges are paid for the items kept in stock $=\frac{c I_{P} \rho}{T}\left[\frac{D T^{2}-P M^{2}}{2}\right]$

2. $M \leq T$ as shown in Fig. 2

In this case, interest charges are paid for the items kept in stock $=\frac{c I_{P} D(T-M)^{2}}{2 T}$

3. $N \leq T \leq M$

No interest charges are paid for the items kept in stock.

$4 \quad 0 \leq T \leq N$ similar as case-2

No interest charges are paid for the items kept in stock 


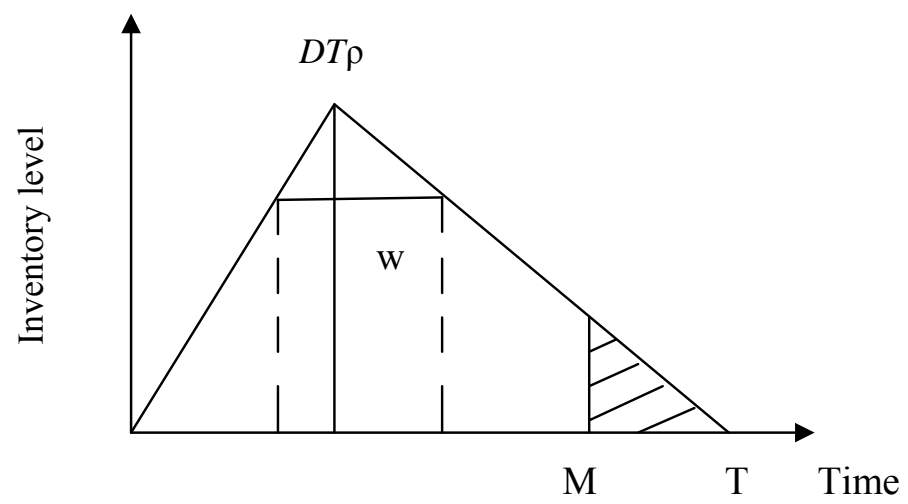

Fig. 2. The inventory level and total accumulation of interest payable when $M \leq T \leq \frac{P M}{D}$ (4) According to assumption (8), interest earned per year can be obtained as follows:

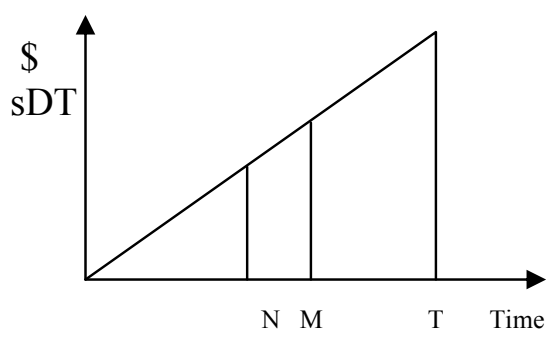

Fig. 3. The total accumulation of interest earned when $M \leq \frac{P M}{D} \leq T$ or $M \leq T \leq \frac{P M}{D}$

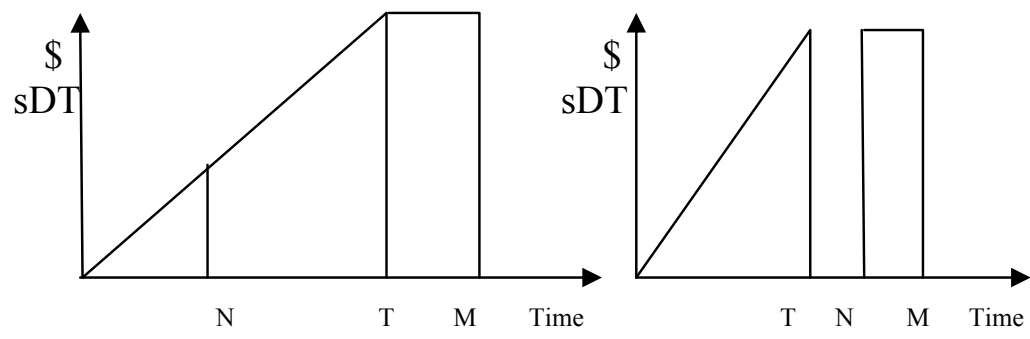

Fig. 4. The total accumulation of Fig. 5. The total interest earned when $N \leq T \leq M$ accumulation of interest When $T \leq N$

1. $M \leq \frac{P M}{D} \leq T$

Annual interest earned $=\frac{\operatorname{sIeD}\left(M^{2}-N^{2}\right)}{2 T}$

2. $M \leq T \leq \frac{P M}{D}$ as shown in Fig 3

Annual interest earned $=\frac{S I_{e} D\left(M^{2}-N^{2}\right)}{2 T}$

3. $N \leq T \leq M$ as shown in Fig. 4

Annual interest earned $=S I_{e}\left[\frac{(D N+D T)(T-N)}{2}+D T(M-T)\right] / T=\frac{S I_{e} D\left(2 M T-N^{2}-T^{2}\right)}{2 T}$

4. $0<T \leq N$ as shown in Fig. 5

Annual interest earned $=\frac{S I_{e} D T(M-N)}{T}$

From the above argument the annual total relevant cost for the retailer can be expressed as $T R C(T)=$ ordering cost + stock-holding cost + interest payable - interest earned.

We show that the annual total relevant cost, $T R C(T)$ is given by

$$
T R C(T)=\left\{\begin{array}{cccc}
T R C_{1}(T) & \text { if } & T \geq \frac{P M}{D} & \text { (a) } \\
T R C_{2}(T) & \text { if } & M \leq T \leq \frac{P M}{D} & \text { (b) } \\
T R C_{3}(T) & \text { if } & N \leq T \leq M & \text { (c) } \\
T R C_{4}(T) & \text { if } & \frac{W}{D \rho} \leq T \leq N & \text { (d) } \\
T R C_{5}(T) & \text { if } & 0<T \leq \frac{W}{D \rho} & \text { (e) }
\end{array}\right.
$$

where 
$T R C_{1}(T)=\frac{A}{T}+\frac{k(D T \rho-W)^{2}}{2 D T}+\frac{h w(D T(1+\rho)-W)}{2 D T}+c I_{P} \rho\left(D T^{2}-P M^{2}\right)-\frac{\operatorname{sIeD}\left(M^{2}-N^{2}\right)}{2 T}$,

$T R C_{2}(T)=\frac{A}{T}+\frac{k(D T \rho-W)^{2}}{2 D T}+\frac{h w(D T(1+\rho)-W)}{2 D T}+\frac{c I_{P} D(T-M)^{2}}{2 T}-\frac{s I_{e} D\left(M^{2}-N^{2}\right)}{2 T}$,

$T R C_{3}(T)=\frac{A}{T}+\frac{k(D T \rho-W)^{2}}{2 D T}+\frac{h W[D T(1+\rho)-W]}{2 D T}-\frac{s I_{e} D\left(2 M T-N^{2}-T^{2}\right)}{2 T}$

$T R C_{4}(T)=\frac{A}{T}+\frac{k(D T \rho-W)^{2}}{2 D T}+\frac{h W[D T(1+\rho)-W]}{2 D T}-\frac{s I_{e} D T(M-N)}{T}$,

and

$T R C_{5}(T)=\frac{A}{T}+\frac{D T h \rho}{2}-s I_{e} D(M-N)$.

SinceTRC $\left(\frac{P M}{D}\right)=T R C_{2}\left(\frac{P M}{D}\right), T R C_{2}(M)=T R C_{3}(M), T R C_{3}(N)=T R C_{4}(N)$ and

$T R C_{4}\left(\frac{w}{D \rho}\right) \neq T R C_{5}\left(\frac{w}{D \rho}\right), T R C(T)$ is continuous except at $T=\frac{W}{D \rho}$ Furthermore, we have $T R C_{4}(T) \geq$ $T R C_{5}(T)$ for all $T>0$.

$T R C_{1}^{\prime}(T)=\frac{-\left[2 A+\frac{w^{2}}{D}(k-h)-c I_{P}(P-D) M^{2}-\operatorname{sIeD}\left(M^{2}-N^{2}\right)\right]}{2 T^{2}}+\frac{D \rho\left(k \rho+c I_{P}\right)}{2}$

$T R C_{1}^{\prime \prime}(T)=\frac{2 A+\frac{w^{2}}{D}(k-h)-c I_{P}(P-D) M^{2}-\operatorname{sIeD}\left(M^{2}-N^{2}\right)}{T^{3}}>0$

$T R C_{2}^{\prime}(T)=\frac{-\left[2 A+\frac{W^{2}}{D}(k-h)+D\left\{M^{2}\left(c I_{p}-s I_{e}\right)+N^{2} s I_{e}\right\}\right]}{2 T^{2}}+\frac{D\left(K \rho^{2}+c I_{p}\right)}{2}$

$T R C_{2}^{\prime \prime}(T)=\frac{2 A+\frac{W^{2}}{D}(k-h)+D\left[M^{2}\left(c I_{p}-s I_{e}\right)+N^{2} s I_{e}\right]}{T^{3}}>0$

$T R C_{3}^{\prime}(T)=\frac{-\left[2 A+\frac{W^{2}}{D}(k-h)+s D N^{2} I_{e}\right]}{2 T^{2}}+\frac{D\left(k \rho^{2}+s I_{e}\right)}{2}$

$T R C_{3}^{\prime \prime}(T)=\frac{2 A+\frac{W^{2}}{D}(k-h)+s D N^{2} I_{e}}{T^{3}}>0$

$T R C_{4}^{\prime}(T)=\frac{-\left[2 A+\frac{W^{2}}{D}(k-h)\right]}{2 T^{2}}+\frac{k D \rho^{2}}{2}$

$\operatorname{TRC}_{4}^{\prime \prime}(T)=\frac{2 A+\frac{W^{2}}{D}(k-h)}{T^{3}}>0$ 
$T R C_{5}^{\prime}(T)=\frac{-2 A}{2 T^{2}}+\frac{D h \rho}{2}$

$T R C_{5}^{\prime \prime}(T)=\frac{2 A}{T^{3}}>0$

Eqs. $(3 B),(4 C),(5 D),(6 E)$ imply that $T R C_{2}(T), T R C_{3}(T), T R C_{4}(T)$ and $T R C_{5}$ are convex on $T>$ 0 . However, $T R C_{1}(T)$ is convex on $T>0$ if $2 A+\frac{w^{2}}{D}(k-h)-c I_{P}(P-D) M^{2}-s I e D\left(M^{2}-\right.$ $\left.N^{2}\right)>0$. Moreover, we have $T R C_{1}^{\prime}\left(\frac{P M}{D}\right)=T R C_{2}^{\prime}\left(\frac{P M}{D}\right), T R C_{2}^{\prime}(M)=T R C_{3}^{\prime}(M), T R C_{3}^{\prime}(N)=$ $T R C_{4}^{\prime}(N)\left(T R C(T)\right.$ is convex) and $T R C_{4}^{\prime}\left(\frac{w}{D \rho}\right) \neq T R C_{5}^{\prime}\left(\frac{w}{D \rho}\right)(T R C(T)$ is piecewise convex, but not convex).

Case. 2 Suppose that $M \geq \frac{W}{D \rho} \geq N$.

If $M \geq \frac{W}{D \rho} \geq N$. Eqs. 1a-e will be modified as

$T R C(T)=\left\{\begin{array}{llcc}T R C_{1}(T) & \text { if } & T \geq \frac{P M}{D} & \text { (a) } \\ T R C_{2}(T) & \text { if } & M \leq T \leq \frac{P M}{D} & \text { (b) } \\ T R C_{3}(T) & \text { if } & \frac{W}{D \rho} \leq T \leq M & \text { (c) } \\ T R C_{6}(T) & \text { if } & N \leq T \leq \frac{W}{D \rho} & \text { (d) } \\ T R C_{5}(T) & \text { if } & 0<T \leq N . & \text { (e) }\end{array}\right.$

When $N \leq T \leq \frac{W}{D \rho}$. the annual total relevant cost, $T R C_{6}(T)$ consists of the following elements:

(1) Annual ordering cost $=\frac{A}{T}$

(2) In this case the order quantity is not larger than retailer's storage capacity. So the retailer will not necessary to rent warehouse to storage items. Hence

Annual stock-holding cost $=\frac{D T h \rho}{2}$

(3) In this case no interest charges are paid for the items kept in stock

(4) Annual interest earned $=\frac{S I_{e} D\left(2 M T-N^{2}-T^{2}\right)}{2 T}$.

Combining above element, we get

$T R C_{6}(T)=\frac{A}{T}+\frac{D T h \rho}{2}-\frac{s I_{e} D\left(2 M T-N^{2}-T^{2}\right)}{2 T}$

Since $T R C_{1}\left(\frac{P M}{D}\right)=T R C_{2}\left(\frac{P M}{D}\right), T R C_{2}(M)=T R C_{3}(M), T R C_{3}\left(\frac{W}{D \rho}\right) \neq T R C_{6}\left(\frac{W}{D \rho}\right)$ and $T R C_{6}(N)=$ $T R C_{5}(N), T R C(T)$ is continuous except at $T=\frac{W}{D \rho}$ Furthermore we have $T R C_{3}(T) \geq T R C_{6}(T)$ for all $T>0$.

$T R C_{6}^{\prime}(T)=-\left[\frac{2 A+s I_{e} D N^{2}}{2 T^{2}}\right]+\frac{D\left(h \rho+s I_{e}\right)}{2}$

$T R C_{6}^{\prime \prime}(T)=\frac{2 A+s I_{e} D N^{2}}{T^{3}}>0$ 
From Eq. (8A) implies that $T R C_{6}(T)$ is convex on $>0$. Moreover we have $T R C_{1}^{\prime}\left(\frac{P M}{D}\right)=$ $T R C_{2}^{\prime}\left(\frac{P M}{D}\right), T R C_{2}^{\prime}(M)=T R C_{3}^{\prime}(M)$ and $T R C_{6}^{\prime}(N)=T R C_{5}^{\prime}(N)$. (TRC $(T)$ is convex) $T R C_{3}^{\prime}\left(\frac{W}{D \rho}\right) \neq$ $T R C_{6}^{\prime}\left(\frac{W}{D \rho}\right)(T R C(T)$ is piecewise convex, but not convex).

Case. 3 Suppose that $\frac{W}{D \rho} \geq M \geq N$.

If $\frac{W}{D \rho} \geq M \geq N$, Eqs. 1a-d will be modified as

$T R C(T)=\left\{\begin{array}{cccc}T R C_{1}(T) & \text { if } & T \geq \frac{P M}{D} & \text { (a) } \\ T R C_{2}(T) & \text { if } & T>\frac{W}{D \rho} & \text { (b) } \\ T R C_{7}(T) & \text { if } & M \leq T \leq \frac{W}{D \rho} & \text { (c) } \\ T R C_{6}(T) & \text { if } & N \leq T \leq M & \text { (d) } \\ T R C_{5}(T) & \text { if } & 0<T \leq N . & \text { (e) }\end{array}\right.$

When $M \leq T \leq \frac{W}{D \rho}$, the annual total relevant $\operatorname{cost}, T R C_{7}(T)$. consist of the following element:

(1) Annual ordering cost $=\frac{A}{T}$

(2) In this case the order quantity is not larger than retailer's storage capacity. So the retailer will not necessary to rent warehouse to storage items. Hence

Annual stock-holding cost $=\frac{D T h \rho}{2}$

(3) Cost of interest charges for the items kept in stock per year $=\frac{c I_{p} D(T-M)^{2}}{2 T}$

(4) Annual interest earned $=s I_{e} D\left(M^{2}-N^{2}\right) / 2 T$.

Combining above elements, we get

$T R C_{7}(T)=\frac{A}{T}+\frac{D T h \rho}{2}+\frac{c I_{p} D(T-M)^{2}}{2 T}-\frac{s I_{e} D\left(M^{2}-N^{2}\right)}{2 T}$

Since $T R C_{1}\left(\frac{P M}{D}\right)=T R C_{2}\left(\frac{P M}{D}\right), T R C_{2}\left(\frac{W}{D \rho}\right) \neq T R C_{7}\left(\frac{W}{D \rho}\right), T R C_{7}(M)=T R C_{6}(M)$ and

$T R C_{6}(N)=T R C_{5}(N), T R C(T)$ is continuous except at $T=\frac{w}{D \rho}$. Furthermore we have $T R C_{2} \geq$ $T R C_{7}(T)$ for all $T>0$ and $T R C_{2}(T)$ will be reduce to $T R C_{7}(T)$.

$T R C_{7}^{\prime}(T)=-\frac{\left[2 A+D\left\{M^{2}\left(c I_{p}-s I_{e}\right)+N^{2} s I_{e}\right\}\right]}{2 T^{2}}+\frac{D\left(h \rho+c I_{p}\right)}{2}$

$T R C_{7}^{\prime \prime}(T)=\frac{2 A+D\left[M^{2}\left\{c I_{p}-s I_{e}\right\}+N^{2} s I_{e}\right]}{T^{3}}>0$

From eq (10A) implies that $T R C_{7}(T)$ is convex on $T>0$. Moreover we have $T R C_{1}^{\prime}\left(\frac{P M}{D}\right)=$ $T R C_{2}^{\prime}\left(\frac{P M}{D}\right) \quad T R C_{7}^{\prime}(M)=T R C_{6}^{\prime}(M)$ and $T R C_{6}^{\prime}(N)=T R C_{5}^{\prime}(N)\left(T R C(T)\right.$ is convex) $T R C_{2}^{\prime}\left(\frac{W}{D \rho}\right) \neq$ $T R C_{7}^{\prime}\left(\frac{W}{D \rho}\right)(T R C(T)$ is piecewise convex, but not convex).

\section{Determination of the optimal cycle time $T^{*}$}

In this section, we shall determine optimal cycle time for the above three situations under minimizing annual total relevant cost using differentiation 
Case.1 Suppose that $M \geq N \geq \frac{W}{D \rho}$.

Then we can rewrite

$T R C_{1}(T)=\frac{2 A+\frac{w^{2}}{D}(k-h)-c I_{p}(P-D) M^{2}-\operatorname{sIeD}\left(M^{2}-N^{2}\right)}{2 T}+\frac{D T \rho\left(k \rho+c I_{p}\right)}{2}-\frac{(2 k w \rho-h w-h w \rho)}{2}$

Diff. with respect to $T$, We get

$T_{1}^{*}=\sqrt{\frac{2 A+\frac{w^{2}}{D}(k-h)-c I_{p}(P-D) M^{2}-\operatorname{sIe} D\left(M^{2}-N^{2}\right)}{D \rho\left(k \rho+c I_{p}\right)}}$

Therefore

$$
\begin{aligned}
T R C_{1}\left(T_{1}^{*}\right)= & \left\{\sqrt{D \rho\left(k \rho+c I_{p}\right)\left[2 A+\frac{w^{2}}{D}(k-h)-c I_{p}(P-D) M^{2}-\operatorname{sIeD}\left(M^{2}-N^{2}\right)\right]}\right. \\
& \left.-\left[\frac{2 k w \rho-h w-h w \rho}{2}\right]\right\}
\end{aligned}
$$

Similarly we can derive $T R C_{2}(T)$ with help of derivatives as follows:

$$
T R C_{2}(T)=\frac{2 A+\frac{W^{2}}{D}(k-h)+D M^{2}\left(c I_{P}-s I_{e}\right)+s I_{e} D N^{2}}{2 T}+\frac{D T\left(k \rho^{2}+c I_{p}\right)}{2}
$$

Diff. with respect to $T$, We get

$T_{2}^{*}=\sqrt{\frac{2 A+\frac{W^{2}}{D}(k-h)+D M^{2}\left(c I_{p}-s I_{e}\right)+s I_{e} D N^{2}}{D\left(k \rho^{2}+c I_{p}\right)}}$

Therefore

$$
\begin{aligned}
T R C_{2}\left(T_{2}^{*}\right)= & \left\{\sqrt{D\left(k \rho^{2}+c I_{p}\right)\left[2 A+\frac{W^{2}}{D}(k-h)+D M^{2}\left(c I_{p}-s I_{e}\right)+s I_{e} D N^{2}\right]}-\{k W \rho\right. \\
& \left.\left.+c I_{p} D M-\frac{h w(1+\rho)}{2}\right\}\right\}
\end{aligned}
$$

Similarly, we can derive $T R C_{3}(T)$ with help of derivatives as follows:

$$
T R C_{3}(T)=\frac{2 A+\frac{W^{2}}{D}(k-h)+s D N^{2} I_{e}}{2 T}+\frac{D T\left(k \rho^{2}+s I_{e}\right)}{2}
$$

Differentiation with respect of $T$, We get 
$T_{3}^{*}=\sqrt{\frac{2 A+\frac{W^{2}}{D}(k-h)+s D N^{2} I_{e}}{D\left(k \rho^{2}+s I_{e}\right)}}$

Therefore

$$
\begin{aligned}
T R C_{3}\left(T_{3}^{*}\right)= & \left\{\sqrt{D\left(k \rho^{2}+s I_{e}\right)\left[2 A+\frac{W^{2}}{D}(k-h)+s D N^{2} I_{e}\right]}\right. \\
& \left.-\left[k W \rho-\frac{h W(1+\rho)}{2}+s I_{e} D M\right]\right\}
\end{aligned}
$$

Likewise, we can derive $T R C_{4}(T)$ by differentiation

$T R C_{4}(T)=\frac{2 A+\frac{W^{2}}{D}(k-h)}{2 T}+\frac{k D T \rho^{2}}{2}-\left[k W \rho-\frac{h W(1+\rho)}{2}+s D I_{e}(M-N)\right]$

Differentiation with respect to $\mathrm{T}$, we get

$T_{4}^{*}=\sqrt{\frac{2 A+\frac{W^{2}}{D}(k-h)}{k D \rho^{2}}}$

Therefore

$T R C_{4}\left(T_{4}^{*}\right)=\left\{\sqrt{k D \rho^{2}\left[2 A+\frac{W^{2}}{D}(k-h)\right]}-\left[k W \rho-\frac{h W(1+\rho)}{2}+s D I_{e}(M-N)\right]\right\}$

At last we can derive $T R C_{5}(T)$ with the help of differentiation

$T R C_{5}(T)=\frac{A}{T}+\frac{D T h \rho}{2}-s I_{e} D(M-N)$

Differentiation with respect to $T$, we get

$T_{5}^{*}=\sqrt{\frac{2 A}{D h \rho}}$

Therefore

$T R C_{5}\left(T_{5}^{*}\right)=\left[\sqrt{2 A D h \rho}-s D I_{e}(M-N)\right]$

Eq. (11) gives the optimal value of $T^{*}$ for the cases when $T \geq \frac{P M}{D}$ so that $T_{1}^{*} \geq \frac{P M}{D}$. we substitute Eq.(11) into $T_{1}^{*} \geq \frac{P M}{D}$, then we can obtain that

$T_{1}^{*} \geq \frac{P M}{D}$

if and only if $\quad-2 A-\frac{w^{2}}{D}(k-h)-c D M^{2} I_{p}+\operatorname{sIeD}\left(M^{2}-N^{2}\right)+\left(\frac{P M}{D}\right)^{2} D\left(k \rho^{2}+c I_{P}\right) \leq 0$ 
Similarly Eq (13) gives the optimal value of $T^{*}$ for the cases when $\frac{w}{D \rho} \leq T \leq \frac{P M}{D}$ so that $\frac{w}{D \rho} \leq T_{2}^{*} \leq$ $\frac{P M}{D}$. We substitute Eq. (13) into $\frac{w}{D \rho} \leq T_{2}^{*} \leq \frac{P M}{D}$, then we can obtain that

$T_{2}^{*} \leq \frac{P M}{D}$

if and only if $\quad-2 A-\frac{w^{2}}{D}(k-h)-c D M^{2} I_{p}+\operatorname{sIeD}\left(M^{2}-N^{2}\right)+\left(\frac{P M}{D}\right)^{2} D\left(k \rho^{2}+c I_{P}\right) \geq 0$

and

$T_{2}^{*} \geq M$ if and only if $\quad-2 A-\frac{W^{2}}{D}(k-h)+D s I_{e}\left(M^{2}-N^{2}\right)+D M^{2} k \rho^{2} \leq 0$

Similarly Eq (15) gives the optimal value of $T^{*}$ for the cases when $N \leq T \leq M$ so that $N \leq T_{3}^{*} \leq$ $M$. we substitute Eq. (15) into $N \leq T_{3}^{*} \leq M$, then we can obtain that

$T_{3}^{*} \leq M$ if and only if $\quad-2 A-\frac{W^{2}}{D}(k-h)+D s I_{e}\left(M^{2}-N^{2}\right)+D M^{2} k \rho^{2} \geq 0$

and

$N \leq T_{3}^{*}$

if and only if $\quad-2 A-\frac{W^{2}}{D}(k-h)+D N^{2} k \rho^{2} \leq 0$

Likewise Eq. (17) gives the optimal value of $T^{*}$ for the cases when $W / D \rho<T \leq N$ so that $W / D \rho<T_{4}^{*} \leq N$ we substitute Eq.(17) into $W / D \rho<T_{4}^{*} \leq N$ then we can obtain that

$T_{4}^{*} \leq N$ if and only if $-2 A-\frac{W^{2}}{D}(k-h)+D N^{2} k \rho^{2} \geq 0$

and $\frac{W}{D \rho} \leq T_{4}^{*}$ if and only if $\quad-2 A+h \frac{W^{2}}{D} \leq 0$

Finally Eq (19) gives the optimal values of $T^{*}$ for the case when $T \leq W / D \rho$ so that $T_{5}^{*} \leq W / D \rho$ we subtitute Eq. (19) into $T_{5}^{*} \leq W / D \rho$ then we can obtain that

$T_{5}^{*} \leq W / D \rho$ if and only if $\quad-2 A+h \frac{W^{2}}{D \rho} \geq 0$

Furthermore, we let

$\Delta_{1}=-2 A-\frac{W^{2}}{D}(k-h)-c D M^{2} I_{p}+\operatorname{sIe} D\left(M^{2}-N^{2}\right)+\left(\frac{P M}{D}\right)^{2} D\left(k \rho^{2}+c I_{p}\right)$

$\Delta_{2}=-2 A-\frac{W^{2}}{D}(k-h)+D s I_{e}\left(M^{2}-N^{2}\right)+D M^{2} \rho^{2} k$

$\Delta_{3}=-2 A-\frac{W^{2}}{D}(k-h)+D N^{2} k \rho^{2}$

$\Delta_{4}=-2 A+h \frac{W^{2}}{D}$

$\Delta_{5}=-2 A+\frac{W^{2} h}{D \rho}$

Eqs. (21) - (25) imply that $\Delta_{1} \geq \Delta_{2} \geq \Delta_{5} \geq \Delta_{3} \geq \Delta_{4}$. From above arguments we know that $T R C_{3}(T)>T R C_{4}(T)$ we can obtain following results.

\section{Theorem 1}

(A) if $\Delta_{1}>0, \Delta_{2}>0, \Delta_{3}>0 . \Delta_{4}>0, \Delta_{5}>0$ then $\operatorname{TRC}\left(T^{*}\right)=\operatorname{TRC}\left(T_{5}^{*}\right)$ and $T^{*}=T_{5}^{*}$

(B) if $\Delta_{1}>0, \Delta_{2}>0, \Delta_{3}>0, \Delta_{4}<0, \Delta_{5}>0$ then $\operatorname{TRC}\left(T^{*}\right)=T R C\left(T_{2}^{*}\right)$ and $T^{*}=T_{4}^{*}$

(C) if $\Delta_{1}>0, \Delta_{2}>0, \Delta_{3}<0, \Delta_{4}<0, \Delta_{5}>0$ then $\operatorname{TRC}\left(T^{*}\right)=\operatorname{TRC}\left(T_{3}^{*}\right)$ and $T^{*}=T_{3}^{*}$

(D) if $\Delta_{1}>0, \Delta_{2}<0, \Delta_{3}<0, \Delta_{4}<0, \Delta_{5}>0$ then $\operatorname{TRC}\left(T^{*}\right)=T R C\left(T_{6}^{*}\right)$ and $T^{*}=T_{2}^{*}$. 
(E) if $\Delta_{1}>0, \Delta_{2}<0, \Delta_{3}<0, \Delta_{4}<0, \Delta_{5}<0$ then $\operatorname{TRC}\left(T^{*}\right)=\operatorname{TRC}\left(T_{2}^{*}\right)$ and $T^{*}=T_{3}^{*}$

(F) if $\Delta_{1}<0, \Delta_{2}>0, \Delta_{3}>0, \Delta_{4}>0, \Delta_{5}>0$ then $\operatorname{TRC}\left(T^{*}\right)=\min \left[\operatorname{TRC}\left(T_{1}^{*}\right), \operatorname{TRC}\left(T_{5}^{*}\right)\right]$

$$
\text { and } T^{*}=T_{1}^{*}, T_{5}^{*}
$$

(G) if $\Delta_{1}<0, \Delta_{2}>0, \Delta_{3}>0, \Delta_{4}<0, \Delta_{5}>0$ then $\operatorname{TRC}\left(T^{*}\right)=\operatorname{TRC}\left(T_{1}^{*}\right)$ and $T^{*}=T_{1}^{*}$

(H) if $\Delta_{1}<0, \Delta_{2}<0, \Delta_{3}<0, \Delta_{4}<0, \Delta_{5}>0$ then $\operatorname{TRC}\left(T^{*}\right)=\operatorname{TRC}\left(T_{1}^{*}\right)$ and $T^{*}=T_{1}^{*}$

(I) if $\Delta_{1}<0, \Delta_{2}<0, \Delta_{3}<0, \Delta_{4}<0, \Delta_{5}>0$ then TRC $\left(T^{*}\right)=\operatorname{TRC}\left(T_{1}^{*}\right)$ and $T^{*}=T_{1}^{*}$

(J) if $\Delta_{1}<0, \Delta_{2}<0, \Delta_{3}<0, \Delta_{4}<0, \Delta_{5}<0$ then $\operatorname{TRC}\left(T^{*}\right)=\operatorname{TRC}\left(T_{1}^{*}\right)$ and $T^{*}=T_{1}^{*}$

Case 2.Suppose that $M \geq \frac{W}{D \rho} \geq N$.

If $M \geq \frac{W}{D \rho} \geq N$, we know $T R C(T)$ as follows from Eqs. 6a-d:

$T R C(T)=\left\{\begin{array}{llcc}T R C_{1}(T) & \text { if } & T \geq \frac{P M}{D} & \text { (a) } \\ T R C_{2}(T) & \text { if } & M>T \frac{P M}{D} & \text { (b) } \\ T R C_{3}(T) & \text { if } & \frac{W}{D \rho} \leq T \leq M & \text { (c) } \\ T R C_{6}(T) & \text { if } & N \leq T \leq \frac{W}{D \rho} & \text { (d) } \\ T R C_{5}(T) & \text { if } & 0<T \leq N . & \text { (e) }\end{array}\right.$

From Eq. (8) we can derive $T R C_{6}(\mathrm{~T})$ with help of derivative as follows:

$T R C_{6}=\frac{2 A+s D N^{2} I_{e}}{2 T}+\frac{D T\left(h \rho+s I_{e}\right)}{2}-s D M I_{e}$

Differentiation with respect to $\mathrm{T}$, we get

$T_{6}^{*}=\sqrt{\frac{2 A+s D N^{2} I_{e}}{D\left(h \rho+s I_{e}\right)}}$

Therefore

$T R C_{6}\left(T_{6}^{*}\right)=\left[\sqrt{D\left(h \rho+s I_{e}\right)\left(2 A+s D N^{2} I_{e}\right)}-s D M I_{e}\right]$

Similarly to the above procedure in case $1 \mathrm{We}$ substitute Eq. (11) into $T_{1}^{*} \geq \frac{P M}{D}$. then we can obtain that

$$
\begin{gathered}
T_{1}^{*} \geq \frac{P M}{D} \\
\text { if and only if } \quad-2 A-\frac{w^{2}}{D}(k-h)-c D M^{2} I_{p}+\operatorname{sIeD}\left(M^{2}-N^{2}\right)+\left(\frac{P M}{D}\right)^{2} D\left(k \rho^{2}+c I_{P}\right) \leq 0
\end{gathered}
$$

Substitute Eq. (13) into $M \leq T_{2}^{*} \leq \frac{P M}{D}$. We can obtain that

$T_{2}^{*} \leq \frac{P M}{D}$ if and only if $\quad-2 A-\frac{w^{2}}{D}(k-h)-c D M^{2} I_{p}+\operatorname{sIe} D\left(M^{2}-N^{2}\right)+\left(\frac{P M}{D}\right)^{2} D\left(k \rho^{2}+c I_{P}\right) \geq 0$ and

$T_{2}^{*} \geq M$ if and only if $\quad-2 A-\frac{W^{2}}{D}(k-h)+D s I_{e}\left(M^{2}-N^{2}\right)+D M^{2} k \rho^{2} \leq 0$

Substituting Eq. (15) into $\frac{W}{D \rho}<T_{3}^{*} \leq M$. then we can obtain that

$T_{3}^{*} \leq M$ if and only if $\quad-2 A-\frac{W^{2}}{D}(k-h)+D s I_{e}\left(M^{2}-N^{2}\right)+D M^{2} k \rho^{2} \geq 0$

and 
$W / D \rho<T_{3}^{*}$ if and only if $\quad-2 A+\frac{W^{2}}{D \rho^{2}}\left(h \rho^{2}+s I_{e}\right)-s D N^{2} I_{e}<0$

Substituting Eq. (26) into $N \leq T_{6}^{*} \leq W / D \rho$, then we can obtain that $T_{6}^{*} \leq W / D \rho$ if and only if $\quad-2 A+\frac{W^{2}}{D \rho^{2}}\left(h \rho+s I_{e}\right)-s D N^{2} I_{e} \geq 0$

and

$N \leq T_{6}^{*}$ if and only if $\quad-2 A+D N^{2} h \rho \leq 0$.

Substituting Eq. (19) into $T_{5}^{*} \leq N$,then we can obtain that

$T_{5}^{*} \leq N$ if and only if $\quad-2 A+D N^{2} h \rho \geq 0$.

Furthermore, we let

$\Delta_{6}=-2 A+\frac{W^{2}}{D \rho^{2}}\left(h \rho^{2}+s I_{e}\right)-s D N^{2} I_{e}$

$\Delta_{7}=-2 A+\frac{W^{2}}{D}\left(\frac{h}{\rho}+\frac{s I_{e}}{\rho^{2}}\right)-s D N^{2} I_{e}$

and

$\Delta_{8}=-2 A+D h \rho N^{2}$

Eqs. (28) - (30) Imply that $\Delta_{1} \geq \Delta_{2} \geq \Delta_{8} \geq \Delta_{7} \geq \Delta_{6}$ From above argument. We can obtain following results.

Theorem 2: Suppose that $M \geq \frac{W}{D \rho} \geq N$, then

(a) if $\Delta_{1}>0, \Delta_{2}>0, \Delta_{6}>0, \Delta_{7}>0, \Delta_{8}>0$. then $\operatorname{TRC}\left(T^{*}\right)=\operatorname{TRC}\left(T_{5}^{*}\right)$ and $T^{*}=T_{5}^{*}$.

(b) if $\Delta_{1}>0, \Delta_{2}>0, \Delta_{6}<0, \Delta_{7}>0, \Delta_{8}>0$, then TRC $\left(T^{*}\right)=\operatorname{TRC}\left(T_{3}^{*}\right)$ and $T^{*}=T_{3}^{*}$.

(c) if $\Delta_{1}>0, \Delta_{2}>0, \Delta_{6}<0, \Delta_{7}>0, \Delta_{8}<0$ then $\operatorname{TRC}\left(T^{*}\right)=\operatorname{TRC}\left(T_{6}^{*}\right)$ and $T^{*}=T_{6}^{*}$

(d) if $\Delta_{1}>0, \Delta_{2}<0, \Delta_{6}<0, \Delta_{7}>0, \Delta_{8}<0$ then $\operatorname{TRC}\left(T^{*}\right)=\operatorname{TRC}\left(T_{2}^{*}\right)$ and $T^{*}=T_{2}^{*}$

(e) if $\Delta_{1}>0, \Delta_{2}<0, \Delta_{6}<0, \Delta_{7}<0, \Delta_{8}<0$ then TRC $\left(T^{*}\right)=\operatorname{TRC}\left(T_{1}^{*}\right)$ and $T^{*}=T_{3}^{*}$

(f) if $\Delta_{1}>0, \Delta_{2}>0, \Delta_{6}<0, \Delta_{7}<0, \Delta_{8}<0$ then $\operatorname{TRC}\left(T^{*}\right)=\min \left[\operatorname{TRC}\left(T_{1}^{*}\right), \operatorname{TRC}\left(T_{5}^{*}\right)\right]$ and $T^{*}=T_{1}^{*}, T_{5}^{*}$

(g) if $\Delta_{1}<0, \Delta_{2}>0 \Delta_{6}>0, \Delta_{7}<0, \Delta_{8}>0$ then $\operatorname{TRC}\left(T^{*}\right)=\operatorname{TRC}\left(T_{1}^{*}\right)$ and $T^{*}=T_{1}^{*}$

(h) if $\Delta_{1}<0, \Delta_{2}>0 \Delta_{6}<0, \Delta_{7}>0, \Delta_{8}>0$ then TRC $\left(T^{*}\right)=\operatorname{TRC}\left(T_{1}^{*}\right)$ and $T^{*}=T_{1}^{*}$

(i) if $\Delta_{1}<0, \Delta_{2}<0, \Delta_{6}<0, \Delta_{7}>0, \Delta_{8}>0$ then $\operatorname{TRC}\left(T^{*}\right)=\operatorname{TRC}\left(T_{1}^{*}\right)$ and $T^{*}=T_{1}^{*}$.

(j) if $\Delta_{1}<0, \Delta_{2}<0, \Delta_{6}<0, \Delta_{7}<0, \Delta_{8}<0$ then $\operatorname{TRC}\left(T^{*}\right)=\operatorname{TRC}\left(T_{1}^{*}\right)$ and $T^{*}=T_{1}^{*}$.

Case 3: Suppose that $\frac{W}{D \rho} \geq M \geq N$.

If $\frac{W}{D \rho} \geq M \geq N$, we know $\operatorname{TRC}(T)$ as follow From Eqs. 8a-d.

$T R C(T)=\left\{\begin{array}{cccc}T R C_{1}(T) & \text { if } & T \geq \frac{P M}{D} & \text { (a) } \\ T R C_{2}(T) & \text { if } & \frac{W}{D \rho} \leq T \leq \frac{P M}{D} & \text { (b) } \\ T R C_{7}(T) & \text { if } & M \leq T \leq \frac{W}{D \rho} & \text { (c) } \\ T R C_{6}(T) & \text { if } & N \leq T \leq M & \text { (d) } \\ T R C_{5}(T) & \text { if } & 0<T \leq N . & \text { (e) }\end{array}\right.$

From EQ. (10) we can derive $T R C_{7}(T)$ with the help of derivative a follows:

$T R C_{7}(T)=\frac{2 A+c I_{p} D M^{2}-s I_{e} D\left(M^{2}-N^{2}\right)}{2 T}+\frac{D T\left(h \rho+c I_{p}\right)}{2}-c D M I_{p}$

Differentiation with respect to $T$, We get 


$$
T_{7}^{*}=\sqrt{\frac{2 A+D\left[M^{2}\left(c I_{p}-s I_{e}\right)+N^{2} s I_{e}\right]}{D\left(h \rho+c I_{p}\right)}}
$$

Therefore,

$T R C_{7}\left(T_{7}^{*}\right)=\left\{\sqrt{D\left(h \rho+c I_{p}\right)\left[2 A+D\left[M^{2}\left(c I_{p}-s I_{e}\right)+N^{2} s I_{e}\right]\right]}-c D M I_{p}\right\}$

Similar to above procedures in cases 1 and cases 2, we substitute Eq. (11) into $T_{1}^{*}>\frac{P M}{D}$. then we can

$$
T_{1}^{*} \geq \frac{P M}{D}
$$

if and only if $\quad-2 A-\frac{w^{2}}{D}(k-h)-c D M^{2} I_{p}+\operatorname{sIeD}\left(M^{2}-N^{2}\right)+\left(\frac{P M}{D}\right)^{2} D\left(k \rho^{2}+c I_{k}\right) \leq 0$

Substitute Eq. (13) into $\frac{w}{D \rho} \leq T_{2}^{*} \leq \frac{P M}{D}$ then we can obtain that

$T_{2}^{*} \leq \frac{P M}{D}$ if and only if $-2 A-\frac{w^{2}}{D}(k-h)-c D M^{2} I_{p}+\operatorname{sIe} D\left(M^{2}-N^{2}\right)+\left(\frac{P M}{D}\right)^{2} D\left(k \rho^{2}+c I_{k}\right) \geq 0$ and

$T_{2}^{*}>W / D \rho$ if and only if $-2 A+\frac{W^{2}}{D \rho^{2}}\left(h \rho^{2}+c I_{P}\right)-D\left[M^{2}\left(c I_{P}-s I_{e}\right)+N^{2} s I_{e}\right] \leq 0$

Substituting Eq. (31) into $M \leq T_{7}^{*} \leq W / D \rho$ then we can obtain that

$T_{7}^{*} \leq W / D \rho$ if and only if $-2 A+\frac{W^{2}}{D \rho^{2}}\left(h \rho+c I_{p}\right)-D\left[M^{2}\left(c I_{p}-s I_{e}\right)+N^{2} s I_{e}\right] \geq 0$

And

$M \leq T_{7}^{*}$ if and only if $-2 A+s I_{e} D\left(M^{2}-N^{2}\right)+D M^{2} h \rho \leq 0$

Substituting Eq. (26) into $N \leq T_{6}^{*} \leq M$, then we can obtain that

$T_{6}^{*} \leq M$ if and only if $-2 A+s I_{e} D\left(M^{2}-N^{2}\right)+D M^{2} h \rho \geq 0$

and $N \leq T_{6}^{*}$ if and only if $-2 A+D N^{2} h \rho \leq 0$.

Substituting Eq.(19) into $T_{5}^{*} \leq N$ then we can obtain that

$T_{5}^{*} \leq N$ if and only if $\quad-2 A+D N^{2} h \rho \geq 0$. Furthermore, we let

$$
\begin{gathered}
\Delta_{9}=-2 A+\frac{W^{2}}{D \rho^{2}}\left(h \rho^{2}+c I_{P}\right)-D\left[M^{2}\left(c I_{P}-s I_{e}\right)+N^{2} s I_{e}\right] \\
\Delta_{10}=-2 A+\frac{W^{2}}{D \rho^{2}}\left(h \rho+c I_{p}\right)-D\left[M^{2}\left(c I_{p}-s I_{e}\right)+N^{2} s I_{e}\right] \\
\Delta_{11}=-2 A+s I_{e} D\left(M^{2}-N^{2}\right)+D M^{2} h \rho
\end{gathered}
$$

Eqs. (33) - (35) imply that $\Delta_{1} \geq \Delta_{10} \geq \Delta_{9} \geq \Delta_{11} \geq \Delta_{8}$ From above arguments, we can obtain following results.

\section{Theorem 3}

(a) if $\Delta_{1}>0, \Delta_{9}>0, \Delta_{10}>0, \Delta_{11}>0, \Delta_{8}>0$ then $\operatorname{TRC}\left(T^{*}\right)=\operatorname{TRC}\left(T_{5}^{*}\right)$ and $T^{*}=T_{5}^{*}$

(b) if $\Delta_{1}>0, \Delta_{9}<0, \Delta_{10}>0, \Delta_{11}>0, \Delta_{8}>0$ then $\operatorname{TRC}\left(T^{*}\right)=\operatorname{TRC}\left(T_{2}^{*}\right)$ and $T^{*}=T_{2}^{*}$

(c) if $\Delta_{1}>0, \Delta_{9}<0, \Delta_{10}>0, \Delta_{11}>0, \Delta_{8}<0$ then TRC $\left(T^{*}\right)=T R C\left(T_{6}^{*}\right)$ and $T^{*}=T_{6}^{*}$

(d) if $\Delta_{1}>0, \Delta_{9}<0, \Delta_{10}>0, \Delta_{11}<0, \Delta_{8}<0$ then $\operatorname{TRC}\left(T^{*}\right)=\operatorname{TRC}\left(T_{7}^{*}\right)$ and $T^{*}=T_{7}^{*}$

(e) if $\Delta_{1}>0, \Delta_{9}<0, \Delta_{10}<0, \Delta_{11}<0, \Delta_{8}<0$ then TRC $\left(T^{*}\right)=\operatorname{TRC}\left(T_{2}^{*}\right)$ and $T^{*}=T_{2}^{*}$

(e) if $\Delta_{1}<0, \Delta_{9}>0, \Delta_{10}>0, \Delta_{11}>0, \Delta_{8}>0$ then $\operatorname{TRC}\left(T^{*}\right)=\min \left[\operatorname{TRC}\left(T_{1}^{*}\right), \operatorname{TRC}\left(T_{5}^{*}\right)\right]$ and $T^{*}=T_{1}^{*}, T_{5}^{*}$

(f) if $\Delta_{1}<0, \Delta_{9}<0, \Delta_{10}>0, \Delta_{11}>0, \Delta_{8}>0$ then $\operatorname{TRC}\left(T^{*}\right)=\operatorname{TRC}\left(T_{1}^{*}\right)$ and $T^{*}=T_{1}^{*}$

(g) if $\Delta_{1}<0, \Delta_{9}<0, \Delta_{10}>0, \Delta_{11}<0, \Delta_{8}>0$ then TRC $\left(T^{*}\right)=T R C\left(T_{2}^{*}\right)$ and $T^{*}=T_{1}^{*}$

(h) if $\Delta_{1}<0, \Delta_{9}<0, \Delta_{10}<0, \Delta_{11}<0, \Delta_{8}>0$ then $\operatorname{TRC}\left(T^{*}\right)=\operatorname{TRC}\left(T_{1}^{*}\right)$ and $T^{*}=T_{1}^{*}$

(i) if $\Delta_{1}<0, \Delta_{9}<0, \Delta_{10}<0, \Delta_{11}<0, \Delta_{8}<0$ then $\operatorname{TRC}\left(T^{*}\right)=\operatorname{TRC}\left(T_{1}^{*}\right)$ and $T^{*}=T_{1}^{*}$ 


\section{Numerical examples}

Let $A=\$ 600 /$ order, $c=\$ 25 /$ unit, $D=5000$ units $/$ year, $h=\$ 5 /$ unit $/$ year, $I_{p}=\$ 0.10 / \$ /$ year, $I_{e}=\$ 0.08 / \$ /$ year, $M=0.12$ year $N=0.08$ year

\section{Table 1}

The optimal cycle time with various value of $W, k, P$ and $s$

\begin{tabular}{|c|c|c|c|c|c|c|c|c|c|c|c|}
\hline$w$ & $S$ & $P$ & $k$ & $\Delta_{1}$ & $\Delta_{2}$ & $\Delta_{3}$ & $\Delta_{4}$ & $\Delta_{5}$ & $T^{*}$ & $Q^{*}$ & $\operatorname{TRC}\left(T^{*}\right)$ \\
\hline & 50 & 9000 & 10 & $>0$ & $<0$ & $<0$ & $<0$ & $<0$ & $T_{3}^{*}=0.1584$ & 792.00 & 4103.10 \\
\hline & & & 15 & $>0$ & $<0$ & $<0$ & $<0$ & $<0$ & $T^{*}=0.1490$ & 745.00 & 4341.02 \\
\hline & & & 20 & $>0$ & $<0$ & $<0$ & $<0$ & $<0$ & $\begin{array}{l}T_{3}=0.1490 \\
T_{3}^{*}=0.1415\end{array}$ & 707.50 & 4548.71 \\
\hline \multirow{10}{*}{150} & 60 & 10000 & 10 & $>0$ & $<0$ & $<0$ & $<0$ & $<0$ & $T_{3}^{*}=0.1458$ & 729.00 & 4315.98 \\
\hline & & & 15 & $>0$ & $<0$ & $<0$ & $<0$ & $<0$ & $T^{*}=0.1367$ & 683.50 & 4596.19 \\
\hline & & & 20 & $>0$ & $<0$ & $<0$ & $<0$ & $<0$ & $T_{3}^{*}=0.1294$ & 647.00 & 4839.80 \\
\hline & 70 & 11000 & 10 & $>0$ & $<0$ & $<0$ & $<0$ & $<0$ & $T_{3}^{*}=0.1367$ & 687.50 & 4461.90 \\
\hline & & & 15 & $>0$ & $<0$ & $<0$ & $<0$ & $<0$ & $T^{*}=01280$ & 640.00 & 4779.11 \\
\hline & & & 20 & $>0$ & $<0$ & $<0$ & $<0$ & $<0$ & $T_{3}^{*}=0.1211$ & 605.50 & 5058.96 \\
\hline & & & & $\Delta_{1}$ & $\Delta_{2}$ & $\Delta_{6}$ & $\Delta_{7}$ & $\Delta_{8}$ & & & \\
\hline & 50 & 9000 & 10 & $>0$ & $<0$ & $<0$ & $<0$ & $<0$ & $T_{3}^{*}=0.1626$ & 813.00 & 4091.30 \\
\hline & & & 15 & $>0$ & $<0$ & $<0$ & $<0$ & $<0$ & $T^{*}=0.1566$ & 783.00 & 4192.04 \\
\hline & & & 20 & $>0$ & $<0$ & $<0$ & $<0$ & $<0$ & $T_{3}-0.1500$ & 759.00 & 4283.96 \\
\hline \multirow{11}{*}{250} & 60 & 10000 & & -0 & & & & & $x_{3}-0.1510$ & & \\
\hline & 60 & 10000 & 10 & $>0$ & $<0$ & $<0$ & $<0$ & $<0$ & $T_{3}^{*}=0.1495$ & 749.50 & 4276.00 \\
\hline & & & 15 & $>0$ & $<0$ & $<0$ & $<0$ & $<0$ & $T_{3}^{*}=0.1434$ & 717.00 & 4406.23 \\
\hline & & & 20 & $>0$ & $<0$ & $<0$ & $<0$ & $<0$ & $T_{3}^{*}=0.1386$ & 693.00 & 4511.86 \\
\hline & 70 & 11000 & 10 & $>0$ & $<0$ & $<0$ & $<0$ & $<0$ & $T_{3}^{*}=0.1626$ & 700.50 & 4391.04 \\
\hline & & & 15 & $>0$ & $<0$ & $<0$ & $<0$ & $<0$ & $T_{3}^{*}=0.1566$ & 670.50 & 4547.14 \\
\hline & & & 20 & $>0$ & $<0$ & $<0$ & $<0$ & $<0$ & $T_{3}^{*}=0.1518$ & 647.00 & 4666.95 \\
\hline & & & & $\Delta_{1}$ & $\Delta_{9}$ & $\Delta_{10}$ & $\Delta_{11}$ & $\Delta_{8}$ & & & \\
\hline & 50 & 9000 & 10 & $>0$ & $<0$ & $<0$ & $<0$ & $<0$ & $T_{2}^{*}=0.1821$ & 910.50 & 3927.99 \\
\hline & & & 15 & $>0$ & $<0$ & $<0$ & $<0$ & $<0$ & $T_{2}^{*}=0.1780$ & 890.00 & 3972.86 \\
\hline & & & 20 & $>0$ & $<0$ & $<0$ & $<0$ & $<0$ & $T_{2}^{*}=0.1750$ & 875.00 & 4006.75 \\
\hline \multirow{7}{*}{350} & 60 & 10000 & 10 & $>0$ & $<0$ & $<0$ & $<0$ & $<0$ & $T_{2}^{*}=0.1686$ & 843.00 & 4056.41 \\
\hline & & & 15 & $>0$ & $<0$ & $<0$ & $<0$ & $<0$ & $T_{2}^{*}=0.1633$ & 816.50 & 4126.90 \\
\hline & & & 20 & $>0$ & $<0$ & $<0$ & $<0$ & $<0$ & $T^{*}-0.1506$ & 798.00 & 4178.13 \\
\hline & 70 & 11000 & 10 & $>0$ & $<0$ & $<0$ & $<0$ & $<0$ & $T^{*}=01574$ & 787.00 & 4162.95 \\
\hline & & & 15 & $>0$ & $<0$ & $<0$ & $<0$ & $<0$ & $1_{2}-0.15 / 4$ & 758.50 & 425092 \\
\hline & & & 20 & $>0$ & $<0$ & $<0$ & $<0$ & $<0$ & $T_{2}^{*}=0.1517$ & 73900 & 4314.42 \\
\hline & & & & & & & & & $T_{2}^{*}=0.1478$ & & \\
\hline
\end{tabular}

From the results of Table1, we can observe the optimal cycle time, optimal order quantity and optimal relevant cost with various parameters of $w, k, s$ and $P$, respectively. The following inferences can be made,

1. The optimal cycle time for the retailer will be decrease as replenishment rate $P$ increases. The retailer will purchase less quantity, since the replenishment rate is fast enough.

2. The optimal cycle time increases when retailers' storage capacity $W$ increases. It means that the retailer will purchase more quantity.

3. The optimal cycle time will decrease and total relevant cost increases as RW unit stockholding cost $k$ increases. Therefore, the retailer will purchase less. 
4. The optimal cycle time will decrease as the unit selling price increases. It means that the retailer will purchase less to take the benefits of the trade credit more frequently.

\subsection{Effect of changing the inventory model parameters}

The sensitivity analyses are performed by varying different parameters and are given in Table 2, 3, 4, 5 and 6.

Sensitive analyses for various inventory model parameters are as follows and remaining parameters are same as from above example.

Table 2

Sensitivity analysis

\begin{tabular}{lllllcccc}
\hline $\mathrm{A}$ & $\mathrm{W}$ & $\mathrm{S}$ & $\mathrm{P}$ & $\mathrm{K}$ & $\Delta_{\boldsymbol{i}}$ & $\boldsymbol{T}_{\boldsymbol{i}}$ & $\boldsymbol{Q}^{*}$ & $\boldsymbol{T R C}\left(\boldsymbol{T}^{*}\right)$ \\
\hline 700 & 150 & 50 & 9000 & 10 & $\Delta_{1}<0 \Delta_{2}<0 \Delta_{3}<0 \Delta_{4}<0 \Delta_{5}<0$ & $T_{1}=0.2692$ & 1346.00 & 4029.61 \\
800 & 250 & 60 & 10000 & 15 & $\Delta_{1}>0 \Delta_{2}<0 \Delta_{6}<0 \Delta_{7}<0 \Delta_{8}<0$ & $T_{3}=0.2096$ & 1048.00 & 5144.09 \\
900 & 350 & 70 & 11000 & 20 & $\Delta_{1}>0 \Delta_{9}<0 \Delta_{10}<0 \Delta_{11}<0 \Delta_{8}<0$ & $T_{2}=0.1931$ & 965.50 & 6288.63 \\
\hline
\end{tabular}

Table 3

Sensitivity analysis

\begin{tabular}{lllllcccc}
\hline $\mathrm{h}$ & $\mathrm{W}$ & $\mathrm{S}$ & $\mathrm{P}$ & $\mathrm{K}$ & $\Delta_{\boldsymbol{i}}$ & $\boldsymbol{T}_{\boldsymbol{i}}$ & $\boldsymbol{Q}^{*}$ & $\boldsymbol{T R C}\left(\boldsymbol{T}^{*}\right)$ \\
\hline 6 & 150 & 50 & 9000 & 10 & $\Delta_{1}<0 \Delta_{2}<0 \Delta_{3}<0 \Delta_{4}<0 \Delta_{5}<0$ & $T_{1}=0.2434$ & 1217.00 & 3738.98 \\
7 & 250 & 60 & 10000 & 15 & $\Delta_{1}>0 \Delta_{2}<0 \Delta_{6}<0 \Delta_{7}<0 \Delta_{8}<0$ & $T_{3}=0.1844$ & 922.00 & 4440.48 \\
8 & 350 & 70 & 11000 & 20 & $\Delta_{1}>0 \Delta_{9}<0 \Delta_{10}<0 \Delta_{11}<0 \Delta_{8}<0$ & $T_{2}=0.1596$ & 798.00 & 5253.88 \\
\hline
\end{tabular}

Table 4

Sensitivity analysis

\begin{tabular}{lllllcccc}
\hline $\mathrm{c}$ & $\mathrm{W}$ & $\mathrm{S}$ & $\mathrm{P}$ & $\mathrm{K}$ & $\Delta_{\boldsymbol{i}}$ & $\boldsymbol{T}_{\boldsymbol{i}}$ & $\boldsymbol{Q}^{*}$ & $\boldsymbol{T R C}\left(\boldsymbol{T}^{*}\right)$ \\
\hline 30 & 150 & 50 & 9000 & 10 & $\Delta_{1}<0 \Delta_{2}<0 \Delta_{3}<0 \Delta_{4}<0 \Delta_{5}<0$ & $T_{1}=0.2319$ & 1159.50 & 3711.47 \\
35 & 250 & 60 & 10000 & 15 & $\Delta_{1}>0 \Delta_{2}<0 \Delta_{6}<0 \Delta_{7}<0 \Delta_{8}<0$ & $T_{3}=0.1860$ & 930.00 & 4132.98 \\
40 & 350 & 70 & 11000 & 20 & $\Delta_{1}>0 \Delta_{9}<0 \Delta_{10}<0 \Delta_{11}<0 \Delta_{8}<0$ & $T_{2}=0.1591$ & 795.50 & 4645.94 \\
\hline
\end{tabular}

Table 5

Sensitivity analysis

\begin{tabular}{cllllcccc}
\hline $\boldsymbol{I}_{\boldsymbol{e}}$ & $\mathrm{W}$ & $\mathrm{S}$ & $\mathrm{P}$ & $\mathrm{K}$ & $\Delta_{\boldsymbol{i}}$ & $\boldsymbol{T}_{\boldsymbol{i}}$ & $\boldsymbol{Q}^{*}$ & $\boldsymbol{T R C}\left(\boldsymbol{T}^{*}\right)$ \\
\hline 0.05 & 150 & 50 & 9000 & 10 & $\Delta_{1}<0 \Delta_{2}<0 \Delta_{3}<0 \Delta_{4}<0 \Delta_{5}<0$ & $T_{1}=0.2092$ & 1046.00 & 3103.68 \\
0.07 & 250 & 60 & 10000 & 15 & $\Delta_{1}>0 \Delta_{2}<0 \Delta_{6}<0 \Delta_{7}<0 \Delta_{8}<0$ & $T_{3}=0.1916$ & 958.00 & 4159.01 \\
0.09 & 350 & 70 & 11000 & 20 & $\Delta_{1}>0 \Delta_{9}<0 \Delta_{10}<0 \Delta_{11}<0 \Delta_{8}<0$ & $T_{2}=0.1648$ & 824.00 & 4674.57 \\
\hline
\end{tabular}

Table 6

Sensitivity analysis

\begin{tabular}{lllllcccc}
\hline $\mathrm{M}$ & $\mathrm{W}$ & $\mathrm{S}$ & $\mathrm{P}$ & $\mathrm{K}$ & $\Delta_{\boldsymbol{i}}$ & $\boldsymbol{T}_{\boldsymbol{i}}$ & $\boldsymbol{Q}^{*}$ & $\boldsymbol{T R C}\left(\boldsymbol{T}^{*}\right)$ \\
\hline 0.15 & 150 & 50 & 9000 & 10 & $\Delta_{1}<0 \Delta_{2}<0 \Delta_{3}<0 \Delta_{4}<0 \Delta_{5}<0$ & $T_{3}=0.2125$ & 1062.50 & 3229.52 \\
0.13 & 250 & 60 & 10000 & 15 & $\Delta_{1}>0 \Delta_{2}<0 \Delta_{6}<0 \Delta_{7}<0 \Delta_{8}<0$ & $T_{3}=0.1860$ & 930.00 & 3892.98 \\
0.11 & 350 & 70 & 11000 & 20 & $\Delta_{1}>0 \Delta_{9}<0 \Delta_{10}<0 \Delta_{11}<0 \Delta_{8}<0$ & $T_{2}=0.1655$ & 827.50 & 4839.70 \\
\hline
\end{tabular}

1. It is observed that as $A$ is increased with an increase in values of $w, s, P$ and $k$ results $T^{*}$ and $Q^{*}$ is decreased but $\operatorname{TRC}\left(T^{*}\right)$ increase. The retailer purchased less quantity.

2. When holding cost increases with an increase in values of $w, S, P$ and $k$ results $T^{*}$ and $Q^{*}$ is decreased but $\operatorname{TRC}\left(T^{*}\right)$ increases. The retailer purchased less quantity.

3. As purchasing cost $c$ is increased with increased values of $w, s, P$ and $k$ results $T^{*}$ and $Q^{*}$ is decreased but $\operatorname{TRC}\left(T^{*}\right)$ increases. The retailer purchased less quantity.

4. If $I e$ is increased with increased values of $w, s, P$ and $k$ results $T^{*}$ and $Q^{*}$ is decreased but $\operatorname{TRC}\left(T^{*}\right)$ increases. The retailer purchased less quantity. 
5. A higher value of $M$ along with increased values of $w, s, P$ and $k$ results $T^{*}$ and $Q^{*}$ is decreased but $T R C\left(T^{*}\right)$ increases. The retailer purchased less quantity.

\section{Conclusion}

This paper developed to determine optimal retailer's replenishment policy in EPQ framework. We extends Huang (2006) model by considering finite replenishment rate. We setup three easy-to-use theorems to help the retailer in accurate and rapidly determine the optimal replenishment decisions under minimizing the total cost of inventory system. Numerical results are provided to illustrate the propose solution procedure and to provide managerial insights. (1) The retailer will purchase less quantity, since the replenishment rate is faster enough (2) when retailers storage capacity $W$ increasing. It means that the retailer will purchase more quantity. (3) When RW unit stock-holding cost $k$ is increasing, the retailer will purchase less quantity. (4) When unit selling price increases the optimal cycle time will decrease the retailer will purchase less quantity. Through sensitive analysis, we show managerial decision for retailer.

\section{References}

Abad P.L., \& Jaggi C.K. (2003). A joint approach for setting unit price and the length of the credit period for a seller when end demand is price sensitive. International Journal of Production Economics 83,115-122.

Aggrawal, S.P., \& Jaggi C.K. (1995). Ordering policies of deteriorating items under permissible delay in payments. Journal of Operation Research Society, 46, 658-662.

Benkherouf, L. (1997). A deterministic order level inventory model for deteriorating items with two storage facilities. International Journal of Production Economics, 48(2), 167-175.

Bhunia, A.K., \& Maiti M. (1998). A two warehouse inventory model for deteriorating items with a linear trend in demand and shortages. Journal of the Operation Research Society, 49(3), 287-292.

Chand, S., \& Ward, J. (1987). A note on economic order quantity under conditions of permissible delay in payments. Journal of Operational Research Society, 38, 83-84.

Chang, C.T., Ouyang, L.Y., \& Teng, J.T. (2003). An EOQ model for deteriorating items under supplier credit linked to ordering quantity. Applied Mathematical Modelling, 27, 983-996.

Chang, H.C., \& Dye, C.Y. (2001). An inventory model for deteriorating items with partial backlogging and permissible delay in payments. International Journal of System Science, 32 ,345352.

Chang, H.J., Hung C.H., \& Dye, C.Y. (2001). An inventory model for deteriorating items with linear trend demand under the condition of permissible delay in payments. Production Planning and Control 12, 274-282.

Chen, M. S., \& Chuang, C.C. (1999). An inventory analysis of light buyer's economic order model under trad4 credit. Asia-Pacific Journal of Operational Research, 16 (1) 23-34.

Chung, K.J. (1998). Theorem on the determination of economic order quantity under conditions of permissible delay in payments. Computer and Operational Research, 25, 49-52.

Chung, K.J., \& Huang, T.S. (2005). The Algorithm to the EOQ Model for Inventory control and Trade-credit. Opsearch, 42, 16-27

Chung, K.J., \& Huang, Y.F. (2003). The optimal cycle time for EPQ inventory model under permissible delay in payments. International Journal of Production Economics, 84, 307-318.

Chung K.J., \& Liao J.J. (2004). Lot-sizing decisions under trade credit depending on the ordering quantity, Computer and Operational Research, 31, 909-928.

Goswami, A., \& Chaudhuri, K.S. (1992). An economic order quantity model for the items with two levels of storage for a linear trend in demand. Journal of Operational Research Society, 43, 2 157167.

Goyal, S.K. (1985). Economic order quantity under conditions of permissible delay in payments. Journal of Operation Research Society, 36, 335-338 
Huang, Y.F. (2003). Optimal retailer's ordering policies in the EOQ model under trade credit financing. Journal of Operational Research Society, 54, 1011-1015.

Huang, Y.F. (2006). An Inventory model under two levels of trade credit and limited storage space derived without derivatives. Applied Mathematical Modelling, 30, 418-436.

Huang, Y.F. (2007a). Economic order quantity under conditionally permissible delay in payments. European Journal of Operational Research, 176, 911-924.

Huang Y.F., \& Chung K.J. (2003). Optimal replenishment and payment policies in the EOQ model under cash discount and trade credit. Asia-Pacific Journal of Operation Research, 20,177-190.

Huang, Y. F. (2007b) Optimal retailer's replenishment decisions in the EPQ model under two levels of trade credit policy. European Journal of Operational Research. 176, 1577-1571.

Hwang H., \& Shinn S.W. (1997) Retailer's pricing and lot sizing policy for exponentially deteriorating product under the condition of permissible delay in payments, Computer and Operational Research 24, 539-547

Jamal, A.M.M., Sarker, B.R. \& Wang, S. (1997) an ordering policy for deteriorating items with allowable shortage and permissible delay in payment, Journal of Operation Research Society 48, 826-833

Jamal, A.M.M., Sarker, B.R. \& Wang, S.(2000) Optimal payment time for a retailer under permitted delay of payment by the Wholesaler, International Journal of Production Economics 66, 59- 66

Lee, C.C., \& Hsu, S.L. (2009). A two-warehouse production model for deteriorating inventory items with time-dependent demands. European Journal of Operational Research, 203(2), 593-600.

Liao, H.C., Tsai, C.H., \& Su, C.T. (2000). An inventory model with deteriorating items under inflation when a delay in payment is permissible. International Journal of Production Economics, $63,207-214$.

Liao, J.J. (2007). On an EPQ model for deteriorating items under permissible delay in payments. Applied mathematical modeling, 31, 393-403.

Mondal, B.N., \& Phaujdar, S. (1989b). An inventory model for deteriorating items and stockdependent consumption rate. Journal of Operation Research Society, 40, 483-488.

Mondal, B.N., \& Phaujdar, S. (1989c). A note on an inventory model with stock-dependent consumption rate. Opsearch, 26, 43-46.

Pakkala, T.P.M., \& Achary, K.K. (1992). A deterministic inventory model for deteriorating items with two warehouse and finite replenishment rate. European Journal of Operational Research, 57(1), 71-76.

Salameh M.K., Abbound N.E., El-kassar A.N., \& Ghattas R.E. (2003). Continuous review inventory model with delay in payments. International Journal of Production Economics, 85, 91-95.

Sarker, B.R., Jamal, A.M.M., \& Wang S. $\left(2000_{\mathrm{a}}\right)$. Supply chain model for perishable products under inflation and permissible delay in payment. Computer and Operational Research, 27, 59-75.

Sarker, B.R., Jamal A.M.M.S., \& Wang. S. (2000b). Optimal payment time under permissible delay in payment for products with deteriorating. Production Planning and Control, 11, 380-390.

Shah, N. H. (1993a). Probabilistic time-scheduling model for an exponential decaying inventory when delay in payments is permissible. International Journal of Production Economics, 32, 77-82.

Shah, N. H. (1993b). A lot size model for exponential decaying inventory when delay in payment is permissible. Cashiers du CERO, 35, 115-123.

Shah, V. R., \& Sreehari, M. (1996). An inventory model for a system with multiple storage facility. Opsearch, 2, 96-106.

Sharma, K.V.S. (1987). A deterministic order level model for deteriorating items with two storage facilities. European Journal of Operational Research, 29(1), 70-73.

Teng, J. T., \& Chang, C. T. (2009). Optimal manufacturer's replenishment policies in the EPQ model under two levels of trade credit policy. European Journal of Operational Research. 195, 358-363.

Teng, J. T., \& Goyal, S. K. (2007). Optimal ordering policies for a retailer in a supply chain with upstream and down-stream trade credit. Journal of Operational Research Society, 58, 1252-1255.

Teng, J.T. (2002). On the economic order quantity under conditions of permissible delay in payments. Journal of operation Research society, 53, 915-918. 